OPEN

SUBJECT AREAS:

STRUCTURAL PROPERTIES

METALS AND ALLOYS

Received

14 November 2014

Accepted

22 January 2015

Published

16 March 2015

Correspondence and requests for materials should be addressed to L.S. (Ishao@tamu.edu) or E.F. (efu@pku.edu.

cn)

\section{Sink property of metallic glass free surfaces}

\author{
Lin Shao ${ }^{1,2}$, Engang Fu ${ }^{3}$, Lloyd Price', Di Chen' ${ }^{1}$, Tianyi Chen' ${ }^{1}$ Yongqiang Wang ${ }^{4}$, Guoqiang $\mathrm{Xie}^{5}$ \\ \& Don A. Lucca ${ }^{6}$
}

\begin{abstract}
'Department of Nuclear Engineering, Texas A\&M University, College Station, TX77843, USA, ²Department of Materials Science \& Engineering, Texas A\&M University, College Station, TX 77843, USA, ${ }^{3}$ School of Physics, Peking University, Beijing 100871, P.R. China, ${ }^{4}$ Los Alamos National Laboratory, Los Alamos, New Mexico 87545, USA, ${ }^{5}$ Institute for Materials Research, Tohoku University, Sendai 980-8577, Japan, ${ }^{6}$ School of Mechanical and Aerospace Engineering, Oklahoma State University, Stillwater, Oklahoma 74078, USA.
\end{abstract}

When heated to a temperature close to glass transition temperature, metallic glasses (MGs) begin to crystallize. Under deformation or particle irradiation, crystallization occurs at even lower temperatures. Hence, phase instability represents an application limit for MGs. Here, we report that MG membranes of a few nanometers thickness exhibit properties different from their bulk MG counterparts. The study uses in situ transmission electron microscopy with concurrent heavy ion irradiation and annealing to observe crystallization behaviors of MGs. For relatively thick membranes, ion irradiations introduce excessive free volumes and thus induce nanocrystal formation at a temperature linearly decreasing with increasing ion fluences. For ultra-thin membranes, however, the critical temperature to initiate crystallization is about $100 \mathrm{~K}$ higher than the bulk glass transition temperature. Molecular dynamics simulations indicate that this effect is due to the sink property of the surfaces which can effectively remove excessive free volumes. These findings suggest that nanostructured MGs having a higher surface to volume ratio are expected to have higher crystallization resistance, which could pave new paths for materials applications in harsh environments requiring higher stabilities.

M etallic glasses (MGs) exhibit unique mechanical and chemical properties due to the absence of long range order and grain boundaries ${ }^{1-3}$. The MG phase is metastable and a transition from complete amorphous to partial crystallization occurs when highly correlated atomic movements are allowed. Nucleation and growth of nanocrystals have been observed under heating ${ }^{4}$, ion/electron irradiation ${ }^{5-7}$, bending ${ }^{8}$, indentation ${ }^{9}$, and pressure ${ }^{10}$. One key to induce athermal nanocrystallization is to introduce excessive free volume (FV) whereas positive FV means more open space. FV can be treated as "flowing defects" in MGs, based on the fact that FV contributes to macroscopic atom transport in the free volume mode ${ }^{11-13}$, and that $\mathrm{FV}$ at sufficiently high levels can induce a localized amorphous-to-crystalline transition. The latter resembles crystalline solids in which point defects above critical densities can induce a crystalline-to-amorphous transition ${ }^{14}$.

In crystalline solids a large supersaturation of point defects cannot develop in the presence of high defect sink densities. Various nanostructured crystalline metals having large surface/interface areas were found to have enhanced defect-self-repairing capabilities ${ }^{15-19}$. Can free surfaces of MGs have similar defect sink properties to remove FV? If true, novel structural engineered MGs might open new doors for obtaining higher stability and higher crystallization resistance. MGs in general have relatively low $\mathrm{T}_{\mathrm{g}}$ (glass transition tempereature) which greatly limits their applications. When heated up to a temperature close to $\mathrm{T}_{\mathrm{g}}$, significant crystal nucleation $\operatorname{starts}^{20}$. If heated with concurrent ion irradiation, the temperature for nanocrystallization is even lower (to be shown in the present study). If crystallization resistance can be systematically increased, $\mathrm{MG}$ applications can be further extended into harsh environments. Examples include MG coating of underground oil pipes or spent nuclear containers in deep geological disposal, or even fuel cladding in reactors.

We used in situ transmission electron microscopy (TEM) with concurrent heavy ion irradiation and annealing to study the microstructural changes of $\mathrm{Zr}_{50} \mathrm{Cu}_{35} \mathrm{Al}_{7} \mathrm{Pd}_{5} \mathrm{Nb}_{3} \mathrm{MG}$ membranes prepared by electropolishing. This particular MG was selected due to its high glass-forming ability and unusual plasticity ${ }^{21}$. The experiments were conducted at the IVEM-Tandem National User Facility at Argonne National Laboratory. A $1 \mathrm{MeV}$ Kr beam was introduced into the TEM chamber, bombarding the MG specimens at $30^{\circ}$ off the electron beam direction.

Figure 1A shows the TEM micrographs and selected area diffraction (SAD) patterns obtained from the MG with the irradiation temperature kept at $693 \mathrm{~K}$, which is lower than the $\mathrm{T}_{\mathrm{g}}(710 \mathrm{~K})$ measured from differential 

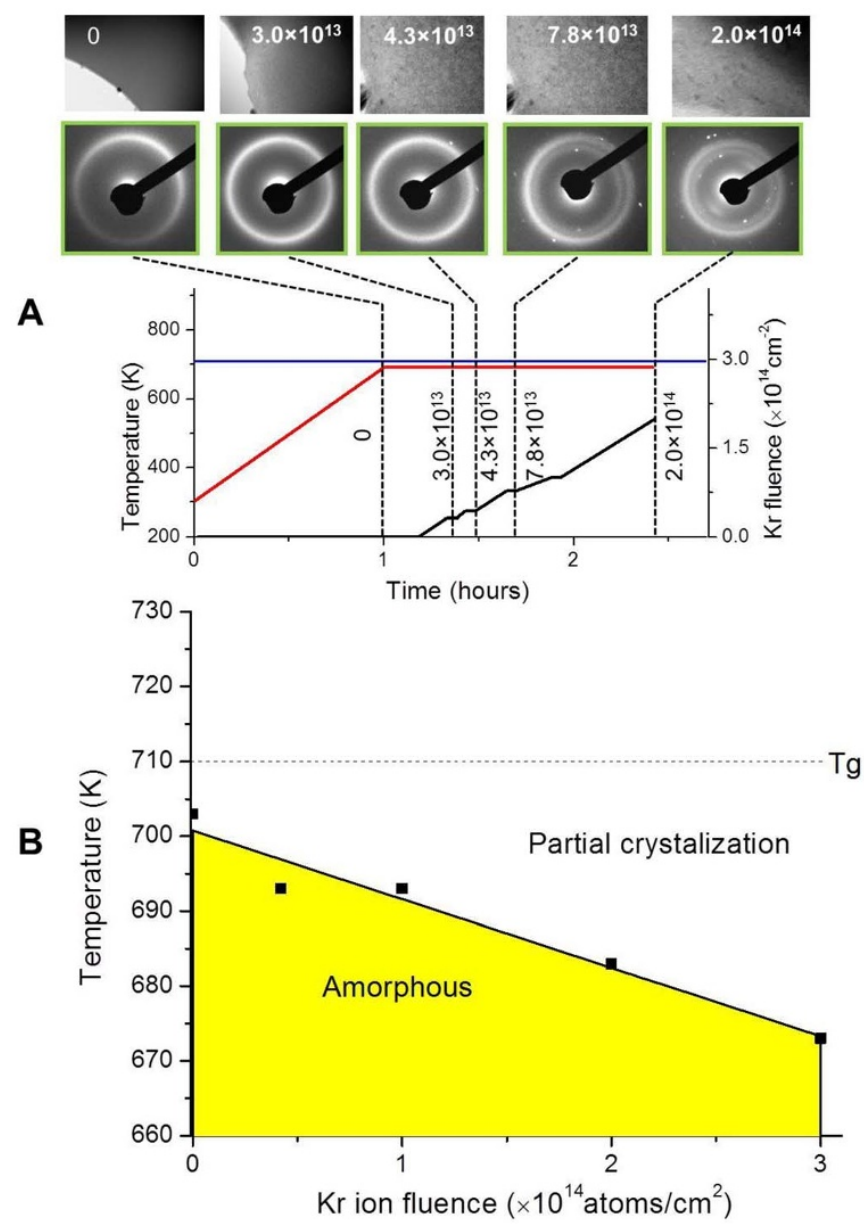

Figure $1 \mid$ Bright field TEM micrographs and corresponding SAD patterns obtained from one irradiation experiment and the summary of the stability boundary for various irradiation conditions. (A) The selected SAD patterns are collected after different fluences of $\mathrm{Kr}$ ion irradiation, as guided by the dash lines. The ion irradiation starts when the annealing temperature reaches $693 \mathrm{~K}$. The red curve shows the time dependent temperature changes and the black line shows the time dependent $\mathrm{Kr}$ ion fluences. The blue line shows the glass transition temperature $\mathrm{T}_{\mathrm{g}}$ measured by using DSC in bulk MG. (B) The yellow region shows the annealing temperature and $\mathrm{Kr}$ ion fluence under which MG membranes still remain complete amorphous structures without nanocrystallization. The dashed line shows $\mathrm{T}_{\mathrm{g}}$. The solid line defining the yellow region is obtained from a linear fit of the solid symbols, which are experimentally determined.

scanning calorimetry (see supplementary Fig. S1). The Kr ion fluence was incrementally increased, and the beam was stopped for a short time period to allow for structural relaxation and TEM imaging. When the $\mathrm{Kr}$ ion fluence reaches $4.3 \times 10^{13} \mathrm{Kr} / \mathrm{cm}^{2}$ and above, nanocrystals appear in the TEM micrographs and white spots appear in the SAD patterns. From a systematic study, Fig. 1B summarizes the temperature vs. $\mathrm{Kr}$ ion fluence required to initiate nanocrystallization. The solid line is a linear fit of the data and is used as the boundary to separate the irradiation stable and unstable regions. Clearly, increasing $\mathrm{Kr}$ ion fluence reduces the temperature required for nanocrystallization.

The most interesting finding is that MG membranes of different thickness exhibit different crystallization resistance. It is typical to find that MG pores created from electropolishing have ultra-thin edges, which appear with relatively brighter contrast in a bright field TEM micrograph. The thickness of this ultra-thin edge is $<15 \mathrm{~nm}$, estimated from electron energy loss spectroscopy. Next to this rim is a relatively thicker region which appears with darker contrast. This thicker region is still electron transparent and its thickness is estimated to be around 100 to $200 \mathrm{~nm}$. As shown in Fig. 2A, at $733 \mathrm{~K}$ and $7.5 \times 10^{13} \mathrm{Kr} / \mathrm{cm}^{2}$ the thick region began to develop nanocrystals, but the ultra-thin region was still completely amorphous. The micrograph observations agree with the SAD results, as shown in Fig. 2B, in which the thick region shows white spots while the ultrathin region still maintains amorphous features. When the temperature is further increased to $813 \mathrm{~K}$ and the fluence to $1.0 \times 10^{14} \mathrm{Kr}$ / $\mathrm{cm}^{2}$, the ultra-thin region begins to develop nanocrystals, as evidenced by the SAD patterns. At higher temperature and higher fluences, nanocrystals in both regions are formed. The study shows that the ultra-thin MG region forms nanocrystals at a temperature which is $120 \mathrm{~K}$ higher than that of the irradiated thick MG, and $103 \mathrm{~K}$ higher than the $\mathrm{T}_{\mathrm{g}}$ of unirradiated $\mathrm{MG}$.

We can use the nucleation rate within the supercooled liquid region as invariance to establish a linkage between various experiments, thus deriving an equation to describe the nanocrystallization temperature shift as a function of ion fluence. Under a steady-state nucleation condition, the nucleation rate, $r$, is given $b^{3}$

$$
r=A D \exp \left(-\frac{\Delta G}{k T}\right)
$$

where $\mathrm{A}$ is a constant, $\mathrm{D}$ is effective diffusivity, $\mathrm{k}$ is the Boltzmann constant, $\mathrm{T}$ is temperature, and $\Delta G$ is the activation energy barrier for forming stable nuclei. The activation energy barrier $\Delta G$ in Eq. [1] can be expressed as $\Delta G=16 \pi \sigma^{3} /\left(3 \Delta G_{l-s}^{2}\right)^{3}$, where $\sigma$ is the interfacial energy and $\Delta G_{l-s}$ is the free energy difference between a liquid state and a crystalline state. The diffusivity $\mathrm{D}$, which originates from the free volume model ${ }^{23,24}$, can be calculated by

$$
D=\left[D_{0} \exp \left(-\frac{E}{k T}\right)\right] \exp \left(\frac{\Delta V_{F V}}{\Delta V_{0}}\right)
$$

where $D_{0}$ is a constant, $E$ is mean migration energy, $\Delta V_{F V}$ is excess free volume, $\Delta V_{0}$ is some activation volume. Assuming a critical $\mathrm{r}$ value is required for nanocrystallization, Eqs. [1] and [2] lead to the following approximation near the $T_{g}$ region:

$$
\Delta T=T_{g}-T \approx T_{g}^{2} \frac{k}{E+\Delta G} \frac{\Delta V_{F V}}{\Delta V_{0}}
$$

where $\Delta T$ is proportional to $\Delta V_{F V}$ and therefore proportional to the $\mathrm{Kr}$ ion fluence. This linear dependence is observed in Fig. 1B. At higher ion fluences if positive FV and negative FV are allowed to dynamically recombine in a manner similar to annihilation of interstitials and vacancies in crystalline solids, excess free volume may saturate and the dependence of $\Delta T$ on ion fluence may deviate from a linear relationship, which is to be verified in a future study.

The above discussion assumes that the irradiation effect on $\Delta G$ is less prominent. The mechanism of $\Delta G$ change is complicated since it is determined by the enthalpy, entropy, and specific heat capacity difference between crystalline and amorphous states ${ }^{25}$. If ion irradiation induces phase segregation, entropy will drop due to the reduced number of microscopic states ${ }^{26}$, thus increasing $\Delta G_{l-s}$. Previous studies on different MGs have observed the agglomeration of nanocrystals of a new phase and subsequent formation of stable nuclei from the agglomeration ${ }^{27}$, but such phase separation was not observed in the present study and will not be a significant driving force for nanocrystallization. Furthermore, introducing excess free volume will decrease the specific heat capacity and thus increase $\Delta G_{l-s}$ although the effect is small.

We conclude that the MG nanocrystal formation observed in the present study is caused by the excessive FV introduced by ion irradiation, instead of localized crystallization along the ion track. Under heavy ion irradiation such as with $\mathrm{Kr}$ ions, the MG membrane can have both scattered displacements and damage cascades forming along the ion track. A damage cascade has a relatively empty core 
A
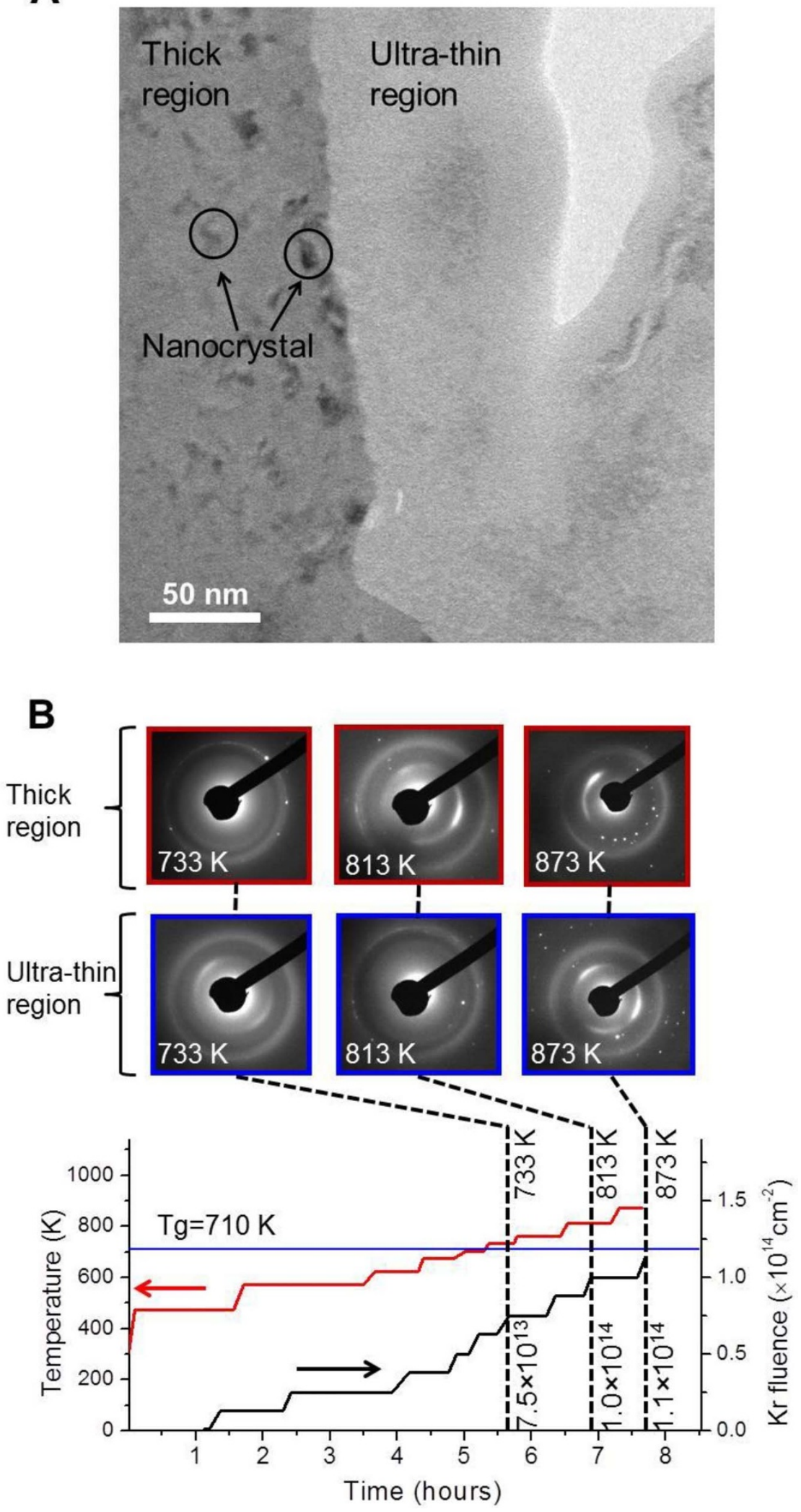

Figure 2 TEM micrograph and SAD patterns obtained from Kr ion irradiated MG membranes. (A) TEM micrograph of the MG membrane irradiated up to $7.5 \times 10^{13} \mathrm{Kr} / \mathrm{cm}^{2}$ and heated up to $733 \mathrm{~K}$. The dark region corresponds to a relatively thicker membrane and the middle bright region corresponds to a relatively thinner membrane. Circles point out typical nanocrystals observed in the thick region, while the ultra-thin region has no nanocrystals observed. (B) The selected SAD patterns collected at different stages of heating and ion irradiation, as guided by the dash lines. The red curve shows temperature changes and the black curve shows $\mathrm{Kr}$ ion fluence changes. The first row of SAD patterns were collected from the thick region, and the second row of SAD patterns were collected from the ultra-thin region, as labeled in (A). For the thick region, white spots appear in all SAD patterns from the thick region; for the ultra-thin region, white spots appear only when the temperature reaches $813 \mathrm{~K}$ and above. 
surrounded by displaced atoms. After a few picoseconds, the kinetic energy of displaced atoms is converted to local heat, leading to thermal spike formation, with temperature in the core high enough to melt MGs. However, the subsequent thermal dissipation is very fast, with a cooling rate over 10 orders of magnitude higher than the critical cooling rate for MG formation, as reported in our previous study $^{22}$. Therefore, the molten region reforms MG. Further evidence that the ion track does not lead to direct crystallization is that the number of nanocrystals formed does not increase with increasing $\mathrm{Kr}$ ion fluence. In one set of experiments performed with simultaneous $\mathrm{Kr}$ ion irradiation and TEM imaging, the number of nanocrystals formed under continuous irradiation was found to saturate. Furthermore, growth of existing nanocrystals into bigger sizes and diminishing of existing nanocrystals were observed (see supplementary Fig. S2). This observation suggests that the nanocrystals require a critical size to reach stability and continuous growth.

The study involved prolonged electron beam analysis. Although previous studies have shown that electron beams can induce nanocrystals, we don't believe this is the case here due to low beam current and low electron energy used. First, electron beam heating was estimated to be about $2 \mathrm{~K}$ (see supplementary), which is ignorable. Second, the TEM electron flux in the present study was two orders of magnitude lower than that used in previous studies which intentionally used a strongly focused electron beam to induce crystalliza- tion $^{5}$. To further evaluate the portion of nanocrystallization caused by the electron beam, we measured the nanocrystallization temperature under concurrent heating and TEM analysis, but without $\mathrm{Kr}$ ion irradiation. This temperature was determined to be $703 \mathrm{~K}$, which is $7 \mathrm{~K}$ below the $\mathrm{T}_{\mathrm{g}}$ (see supplementary Fig. S3). The data was plotted in Fig. $1 \mathrm{~B}$ corresponding to zero $\mathrm{Kr}$ ion fluence. The small temperature difference suggests that the electron beam plays a small, if not ignorable role.

The present study shows that MG is similar to a nanostructured crystalline solid in which free surfaces can serve as defect (FV) sinks. MG free surfaces are expected to be able to remove both positive FV and negative FV, thus leading to higher crystallization resistance. Additional evidence of this FV sink property of MG surfaces is provided through our MD simulations. Due to the complexity of the interatomic potential pairs required to describe a true multi-element MG, the present modeling used a pure amorphous Ni film for simplicity. The Ni amorphous structure is created by first heating a crystalline $\mathrm{Ni}$ film up to $4000 \mathrm{~K}$ and then quenching it down to $1 \mathrm{~K}$ instantly to freeze its amorphous nature. The film is then slowly heated from $1 \mathrm{~K}$ to $600 \mathrm{~K}$ for structural relaxation. At time $\mathrm{t}=0$, the relaxed film is then introduced with $\mathrm{FV}\left(\Delta V_{F V}>0\right.$ by randomly removing $20 \%$ of the atoms within the whole film. For atoms around a removed one, their larger atomic separation distances lead to higher potential energies. Therefore, the FV contained region can be visually
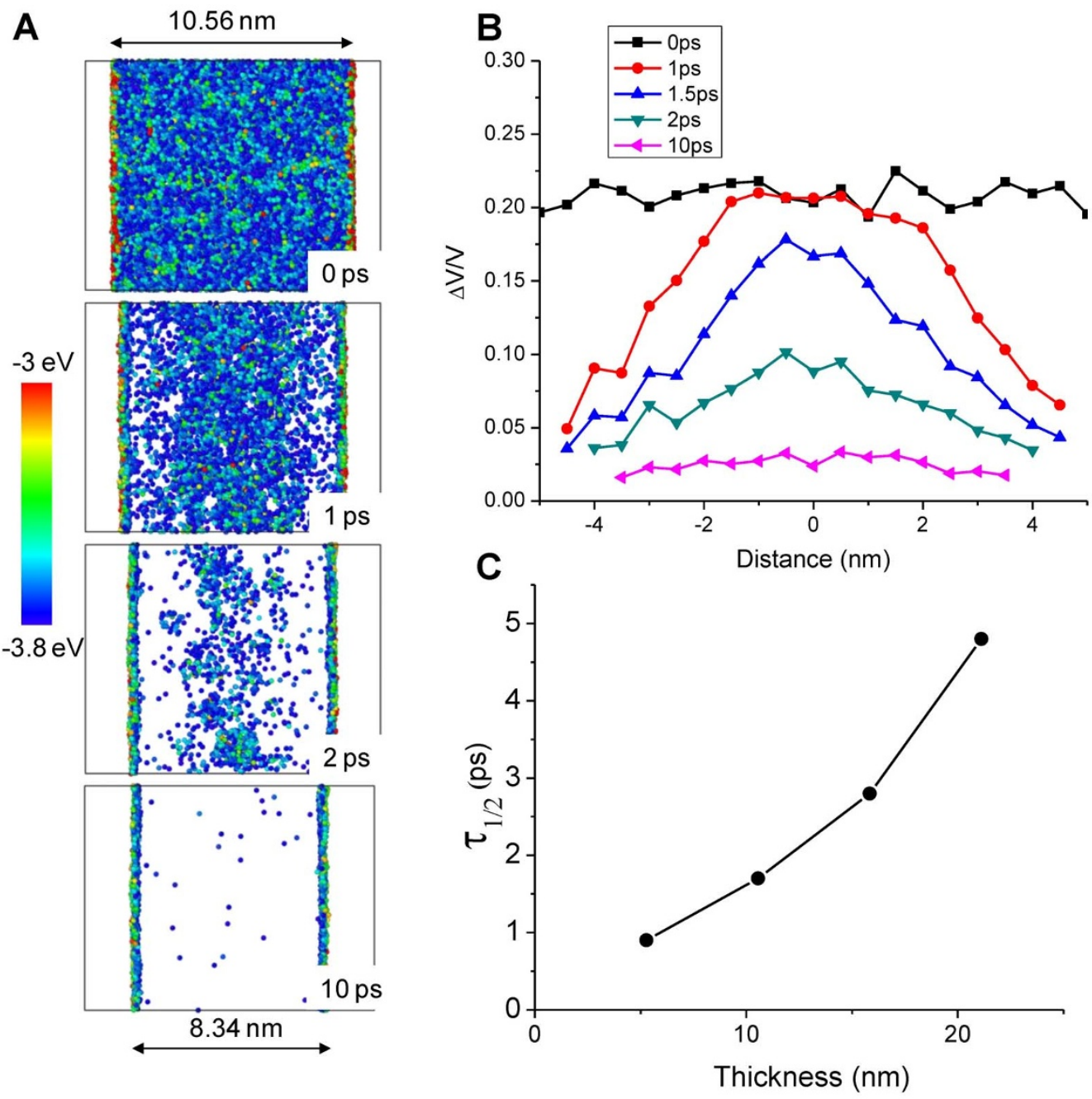

Figure 3 Molecular dynamics simulations of an amorphous Ni film. (A) The plot of atoms having potential energy above $-3.8 \mathrm{eV}$, as a function of annealing time at $600 \mathrm{~K}$. At time $\mathrm{t}=0,20 \%$ atoms are randomly removed to introduce FV. Arrows refer to the film thickness. (B) The plot of $\Delta V / V$ as a function of annealing time, where $V$ is the mean volume occupied by one atom. (C) The plot of required time to reduce $\Delta V / V$ by half in the film center as a function of film thickness. 
mapped by plotting the atoms having potential energies above a certain value $(-3.8 \mathrm{eV})$, as shown in Fig. 3A. With increasing annealing time at $600 \mathrm{~K}, \mathrm{FV}$ is gradually removed, starting first from the film surfaces. At time $t=10 \mathrm{ps}$, the majority of FV is removed. The surface atoms always have higher potential energy. FV removal also leads to shrinkage of the film thickness. Figure $3 \mathrm{~B}$ plots $\mathrm{FV}$ distributions, obtained by calculation of $\Delta V / V$, where $V$ is the mean volume occupied by one $\mathrm{Ni}$ atom in a relaxed amorphous film and $\Delta V$ is the amount of volume increased due to FV introduction. The original box-like FV distribution evolves into a Gaussian like peak at longer times, and eventually the peak diminishes. Such changes can be approximated by diffusion-mediated FV spreading under the boundary condition that excessive FV is fixed to be zero at the two surfaces.

The likelihood of crystallization can be quantitatively represented by time duration when FV is kept above a critical value. We therefore introduce $\tau_{1 / 2}$ as the time required for the film center to reduce down to the half of its original value at $t=0$. Figure $3 \mathrm{C}$ plots the $\tau_{1 / 2}$ as a function of the film thickness. Considerable FV removal in the center occurs when the FV diffusion length becomes comparable to half of the film thickness $d$, as approximated by $d / 2=\sqrt{2 D t}$. This approximation agrees with Fig. 3C in which the data trend suggests $\tau_{1 / 2} \propto d^{2}$. Longer $\tau_{1 / 2}$ in a thicker film gives a higher possibility to develop correlated atomic rearrangements, and the opposite occurs for an ultra-thin film.

The study suggests a path to systematically enhance resistance to crystallization for MGs by using surfaces or interfaces as sinks to remove FV, or as annihilation centers to combine positive and negative FV. Since FV fluctuations can be introduced by thermal annealing or electron/ion irradiation, the proposed structural engineered MGs can have longer lifetime under harsh environments involving higher temperature or particle irradiation. The FV sinks are not necessarily only free surfaces. Interfaces of different MGs can also function as sinks. Interface reactions between different MG systems may reduce MG stability but issues may be alleviated by introducing interfaces of immiscible systems. It is also attractive to introduce multilayered structures with alternatively deposited MG and other advanced highly stable materials such as newly identified ceramics which can withstand high temperature over $1,773 \mathrm{~K}^{28}$.

\section{Methods}

Sample synthesis and TEM specimen preparation. During sample synthesis, a ribbon $\mathrm{Zr}_{50} \mathrm{Cu}_{35} \mathrm{Al}_{7} \mathrm{Pd}_{5} \mathrm{Nb}_{3}$ alloy sample, $1.5 \mathrm{~mm}$ wide and $20 \mu \mathrm{m}$ thick, was fabricated by melting mixtures of pure metal powders and rapid solidification of the melt on a copper roller in an argon atmosphere. A jet electro polisher set up was used to prepare TEM specimens. The polisher reservoir was first filled with a mixture of $75 \%$ methanol $25 \%$ nitric acid to a level that comfortably covered the suction intake of the pump. This mixture was cooled using liquid nitrogen until the temperature was measured to be below $258 \mathrm{~K}$. The sample was then cut to length, $\approx 5 \mathrm{~mm}$, and mounted into the holder. With the setup complete, the sample was repeatedly polished in intervals from 2-10 seconds, inspecting between each polish under an optical microscope, until a hole was seen to develop in the metallic glass sample. This indicated that the sample was electron transparent, and ready for use in the TEM.

Ion irradiation and in situ TEM characterization. The in situ TEM characterization with concurrent ion irradiation was performed by using the IVEM Tandem National User Facility at Argonne National Laboratory (ANL). The angle between the incident electron beam and the ion beam was about 30 degrees. The IVEM Tandem is an intermediate voltage microscope interfaced with a $2 \mathrm{MV}$ ion accelerator. A Kr beam of $1 \mathrm{MeV}$ energy was rastered over the MG specimen to guarantee uniformity. Prior to ion irradiation, specimens were slowly preheated to the designed temperature. All TEM micrographs and diffraction patterns were collected when the $\mathrm{Kr}$ ion beam was off. A movie showing dynamic nanocrystal changes was taken when the $\mathrm{Kr}$ ion beam was on. Position shifting occurred during heating and ion irradiation, therefore position adjustments were always needed and it was difficult to perform characterization on the same spot.

Molecular dynamics simulations. MD simulations were performed by using the LAMMPS $\operatorname{code}^{29}$. An empirical EAM potential was used to describe Ni interatomic interactions ${ }^{30}$. Amorphous structures were obtained by heating up to $4000 \mathrm{~K}$ for $0.5 \mathrm{~ns}$, and cooling down to $1 \mathrm{~K}$ instantly. The structures were then slowly heated from $1 \mathrm{~K}$ to $600 \mathrm{~K}$ to allow for structural relaxation. To create free volume, $20 \%$ of the atoms were randomly selected and removed from the cell. The subsequent structural changes were modeled at a constant temperature of $600 \mathrm{~K}$, under a NVT ensemble. MG membranes of different thickness, 5.3, 10.6, 15.8 and $21.1 \mathrm{~nm}$, were modeled. The thicknesses correspond to 15, 30, 45, 60 unit cell lengths in the starting Ni super cells.

1. Greer, A. L. Metallic glasses. Science 267, 1947-1953 (1995); DOI:10.1126/ science.267.5206.1947.

2. Inoue, A. Stabilization of metallic supercooled liquid and bulk amorphous alloys. Acta Mater. 48, 279-306 (2006); DOI:10.1016/S1359-6454(99)00300-6.

3. Wang, W. H., Dong, C. \& Shek, C. H. Bulk metallic glasses. Mat. Sci. \& Eng. R, Reports 44, 45-89 (2004).

4. Scott, M. G. in Amorphous metallic alloys (ed. F. E. Luborsky), pp 144-168, Butterworths, Boston, 1983.

5. Fu, G. et al. Electron Irradiation Induced Structural Transformation in Metallic Glasses. Script. Mater. 61, 40-43 (2009).

6. Nagase, T. \& Umakoshi, Y. Thermal crystallization and electron irradiation

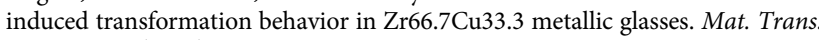
46, 616-621 (2005).

7. Carter, J. et al. Ion irradiation induced nanocrystal formation in amorphous Zr55Cu30Al10Ni5 alloy. Nucl. Instrum. Method. Phys. Rev. B 267, 2827-2831 (2009); DOI:10.1016/j.nimb.2009.05.068.

8. Chen, H., He, Y., Shiflet, G. J. \& Poon, S. J. Deformation-induced nanocrystal formation in shear bands of amorphous alloys. Nature 367, 541-543 (1994); DOI:10.1038/367541a0.

9. Kim, J.-J., Choi, Y., Suresh, S. \& Argon, A. S. Nanocrystallization during nanoindentation of a bulk amorphous metal alloy at room temperature. Science 295, 654-657 (2002); DOI:10.1126/science.1067453.

10. Wang, Z. X., Li, F. Y., Pan, M. X., Zhao, D. Q. \& Wang, W. H. Effects of high pressure on the nucleation of Cu60Zr20Hf10Ti10 bulk metallic glass. J. Alloys and Compounds 388, 262-265 (2005).

11. Turnbull, D. \& Cohen, M. H. Concerning reconstructive transformation and formation of glass. J. Chem. Phys. 29, 1049-1054 (1958); DOI:10.1063/ 1.1744654 .

12. Turnbull, D. \& Cohen, M. H. Free-volume model of the amorphous phase: glass transition. J. Chem. Phys. 34, 120-125 (1961); DOI:10.1063/1.1731549.

13. Beukel, A. \& van den, Sietsma, J. Diffusivity and viscosity during structural relaxation in metallic glasses. Mat. Sci. Eng. A 179/180, 86-90 (1994).

14. Nastasi, M., Mayer, J. W. \& Hirvonen, J. K. Ion-solid interactions: fundamentals and applications. Cambridge University Press, New York, 1996.

15. Nita, N., Schaeublin, R. \& Victoria, M. Impact of irradiation on the microstructure of nanocrystalline materials. J. Nucl. Mater. 329-333, 953 (2004).

16. Chimi, Y. et al. Accumulation and recovery of defects in ion-irradiated nanocrystalline gold. J. Nucl. Mater. 297, 355 (2001).

17. Demkowicz, M. J., Hoagland, R. G. \& Hirth, J. P. Interface structure and radiation damage resistance in $\mathrm{Cu}-\mathrm{Nb}$ multilayer nanocomposites. Phys. Rev. Lett. 100 136102 (2008); DOI:10.1103/PhysRevLett.100.136102.

18. Bai, X.-M., Voter, A. F., Hoagland, R. G., Nastasi, M. \& Uberuaga, B. P. Efficient annealing of radiation damage near grain boundaries via interstitial emission. Science 327, 1631-1634 (2010); DOI:10.1126/science.1183723.

19. Chen, D., Wang, J., Chen, T. \& Shao, L. Defect annihilation at grain boundaries in alpha-Fe. Sci. Rep. 3, 1450 (2013); DOI:10.1038/srep01450.

20. Wang, J. Q. et al. Identification of nanocrystal nucleation and growth in Al85Ni5Y8Co2 metallic glass with quenched-in nuclei. Appl. Phys. Lett. 80, 3319-3321 (2002); DOI:10.1063/1.1476388.

21. Zhu, S., Xie, G., Qin, F., Wang, X. \& Inoue, A. Ni- and Be-free Zr-based bulk metallic glasses with high glass-forming ability and unusual plasticity. J. Mech. Behavior of Biomed. Mat. 13, 166-173 (2012).

22. Myers, M. et al. An experimental and modeling study on the role of damage cascade formation in nanocrystallization of ion-irradiated Ni52.5Nb10Zr15Ti15Pt7.5 metallic glass. Script. Mater. 63, 1045-1048 (2010).

23. Spaepen, F. A microscopic mechanism for steady state inhomogeneous flow in metallic glasses. Acta Metallurgica 25, 407-415 (1977).

24. Rosato, V. \& Cleri, F. A molecular dynamics simulations of the effects of excess free volume on the diffusion in metallic glasses. J. Non-Crystal. Solids 144, 187-195 (1992)

25. Bush, R., Kim, Y. J. \& Johnson, W. L. Thermodynamics and kinetics of the undercooled liquid and the glass transition of the Zr41.2Ti13.8Cu12.5Ni10.0Be22.5 alloy. J. Appl. Phys. 77, 4039-4043 (1995).

26. Inoue, A. High strength bulk amorphous alloys with low critical cooling rate (overview). Mater. Transactions 36, 866-875 (1995).

27. Fu, E. G. et al. Ar-ion-milling-induced structural changes of Cu50Zr45Ti5 metallic glass. Nucl. Instrum. Method. Phys. Res. B 268, 545-549 (2010).

28. Riedel, R., Kleebe, H.-J., Schönfelder, H. \& Aldinger, F. A covalent micro/nanocomposite resistant to high-temperature oxidation. Nature 374, 526-528 (1994); DOI:10.1038/374526a0.

29. Plimpton, S. Fast parallel algorithms for short-range molecular dynamics. J. Comp. Phys. 117, 1-19 (1995)

30. Bonny, G., Pasianot, R. C., Castin, N. \& Malerba, L. Ternary Fe-Cu-Ni many-body potential to model reactor pressure vessel steels: first validation by simulated 
thermal annealing. Phil. Mag. A 89, 3531-3546 (2009); DOI:10.1080/ 14786430903299824

\section{Acknowledgments}

We acknowledge the financial support from the National Science Foundation (USA) through grants no.:1130589 and no.: 1130606.

\section{Author contributions}

L.S. designed the experiments and prepared the manuscript. E.F., L.P. and L.S. performed ion irradiation experiments, D.C. and T.C. performed modeling, Y.W. and D.A.L. performed structural and mechanical characterization, G.X. synthesized specimen. All authors analyzed and discussed results.

\section{Additional information}

Supplementary information accompanies this paper at http://www.nature.com/ scientificreports

Competing financial interests: The authors declare no competing financial interests.

How to cite this article: Shao, L. et al. Sink property of metallic glass free surfaces. Sci. Rep. 5, 8877; DOI:10.1038/srep08877 (2015).

(c) This work is licensed under a Creative Commons Attribution 4.0 International License. The images or other third party material in this article are included in the article's Creative Commons license, unless indicated otherwise in the credit line; if the material is not included under the Creative Commons license, users will need to obtain permission from the license holder in order to reproduce the material. To view a copy of this license, visit http://creativecommons.org/licenses/by/4.0/ 